\title{
Avaliação respiratória em crianças obesas e não obesas
}

Respiratory evaluation in obese

and non-obese children

FisiSenectus. Unochapecó Ano 1, n. 2 - Jul./Dez. 2013 p. $65-72$

\section{Indiamara de Oliveira Flores Dal Magro Silvani}

Mestre em Engenharia Biomédica, professora do curso de Fisioterapia na Unochapecó.

\section{Aline Werlang}

Acadêmica do $9^{\circ}$ período do curso de graduação em Fisioterapia da Unochapecó.

\section{Tuanna Agne}

Acadêmica do $9^{\circ}$ período do curso de graduação em Fisioterapia da Unochapecó.

\section{Resumo}

Introdução: a obesidade é um dos problemas que se encontram associados, direta ou indiretamente, a uma ampla variedade de doenças coletivamente responsáveis por uma porcentagem significativa da morbidade anual nesse país. Objetivos: o presente trabalho teve como objetivo identificar quais as diferenças na força muscular inspiratória e expiratória máximas e o volume de pico máximo de fluxo expiratório. Materiais e métodos: a metodologia utilizada foi a leitura dos resultados no aparelho Peak Flow e Manovacuômetro, nos quais avaliam respectivamente o pico máximo de fluxo expiratório e a força muscular. 0 grupo amostral foi composto por 40 crianças na faixa etária de 8 a 13 anos, sendo divididos em dois grupos (obesos e não obesos). Resultados: nos resultados da avaliação respiratória podem-se perceber diferenças entre as médias na força inspiratória de $14,50 \mathrm{cmH}_{2} \mathrm{O}$, na força expiratória $22,50 \mathrm{cmH}_{2} \mathrm{O}$ e no pico máximo de fluxo expiratório 98,50L/min, sendo essa diferença desfavorável para o grupo de crianças obesas. Para análise dos dados utilizou-se média, desvio padrão e Teste t. Conclusão: pode-se concluir que o grupo de crianças obesas não atingiu o valor esperado para sua altura, ficando distante do grupo de crianças não obesas. As crianças obesas apresentaram redução no pico máximo de fluxo expiratório e redução da força muscular inspiratória e expiratória. A obesidade traz consequências sociais e psicológicas em potencial, a população infantil merece maior enfoque, pois acarreta alterações posturais e psicológicas que poderão perdurar por toda a vida do indivíduo.

\section{Palavras-chave}

Obesidade infantil. Força respiratória. Fisioterapia.

\section{Fisiß̊nectus}




\begin{abstract}
Introduction: obesity is one of the problems which are associated, directly or indirectly, to a wide variety of diseases collectively responsible for a significant percentage of annual morbidity in this country. Objectives: this study had the objective of identify which are the differences in the maximum muscular inspiratory and expiratory strength and the volume of maximum peak of expiratory flow. Materials and methods: the methodology used was the reading of the results on the Peak Flow device and the Vacuum Manometer, which evaluate respectively the maximum peak of expiratory flow and the muscular strength. The sample group was consisted of 40 children ranging in the age from 08 to 13 years old; they were divided in two groups (obese and non-obese). Results: in the results of the respiratory assessment it can be seen differences between the averages in the respiratory strength of $14,50 \mathrm{cmH}_{2} \mathrm{O}$, in the expiratory strength $22,50 \mathrm{cmH}_{2} \mathrm{O}$ and in the maximum peak of expiratory flow $98,50 \mathrm{~L} / \mathrm{min}$, being this difference not favorable to the obese group of children. For data analysis it was used the average, standard deviation and Test t. Conclusion: it can be concluded that the group of obese children has not reached the expected v'alue for their height, getting away from the group on non-obese children. Obese children showed reduction in the maximum peak of respiratory flow and reduction of the inspiratory and expiratory muscular strength. Obesity brings social and psychological consequences in potential; the child population deserves more focus, because it causes postural and psychological alteration that may last for the individual's entire life.
\end{abstract}

\title{
Keywords
}

Childhood obesity. Respiratory strength. Physical therapy.

\section{Introdução}

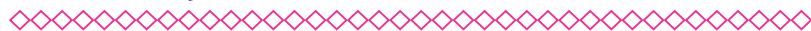

A obesidade é conhecida como o acúmulo de tecido gorduroso, regionalizado em todo o corpo, de origem genética, endócrino-metabólica ou por distúrbios nutricionais ${ }^{1}$.

No Brasil é cada vez mais evidente o aumento de casos de obesidade e sobrepeso nos diferentes seguimentos da população brasileira. Nos últimos anos, nota-se um aumento progressivo de crianças com obesidade. As taxas aumentam 0,5\% ao ano, e em dez anos houve aumento na prevalência de obesidade e sobrepeso em crianças e adolescentes nas regiões nordeste e sudeste de 4,1\% para $13,9 \%{ }^{2}$, tornando-se um problema de saúde pública, pela alta incidência, elevado o número de fatores predisponentes e pelas alterações decorrentes da patologia. A infância é um estágio da vida que merece maior enfoque, pois acarreta alterações posturais e psicológicas que poderão perdurar por toda a vida do indivíduo ${ }^{3}$.

O diagnóstico de sobrepeso e obesidade em crianças recomendado pela Organização Mundial da Saúde (OMS) baseia-se na distribuição de peso para altura, que é a relação entre o peso encontrado e o peso ideal para a altura, sendo por um longo período o único método para diagnosticar alterações nutricionais da infância 4 .

A grande diferença entre obesidade e sobrepeso reside na maior porcentagem de massa corporal com gordura no obeso, ou seja, a expansão dos tecidos magros livres de gordura não foi acompanhada pelo crescimento do tecido adiposo ${ }^{5}$.

A obesidade constitui um fator de risco para outras doenças, podendo trazer alterações na mecânica respiratória causadas principalmente pelo acúmulo de gorduras nas costelas, diafragma e abdômen, levando a um maior consumo de oxigênio para respiração. Consequentemente, contribui de forma direta para a manutenção da obesidade na fase adulta, ocasionando a elevação de doenças associadas à mortalidade, como as cardiovasculares, síndrome metabólica, diabetes, hipertensão e acidentes vasculares cerebrais ${ }^{6,7}$.

Uma das maiores alterações em crianças com obesidade é o comprometimento da função pulmonar e a troca gasosa, pois o tecido adiposo em excesso modifica a mecânica das paredes pulmonares e limita os movimentos do diafragma. A hipoventilação no trato respiratório baixo causa um descompasso entre a ventilação e a perfusão, levando a uma oxigenação arterial abaixo do normal. Este estado de hipoxemia é exacerbado por 
uma necessidade maior de oxigênio, provocada pelos mecanismos respiratórios alterados e pelo aumento de energia requerido pelos movimentos corporais $^{5}$.

A obesidade infantil produz vários efeitos metabólicos adversos, encontra-se igualmente associada a problemas não fatais, mas debilitantes, tais como dificuldade respiratória, problemas musculoesqueléticos, dermatológicos e infertilidade ${ }^{8}$.

Neste contexto, o presente estudo tem por objetivo identificar quais as diferenças na força da musculatura inspiratória e expiratória máxima e do volume de pico máximo de fluxo expiratório de crianças obesas e não obesas do município de Chapecó.

\section{Materiais e métodos}

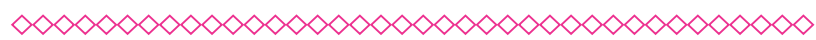

A presente pesquisa apresenta caráter descritivo, comparativo e análise quantitativa dos resultados. Foi realizada com amostra de 40 participantes, sendo divididos em Grupo 1, composto por crianças não obesas, e Grupo 2, por crianças obesas. Nesta amostra, a faixa etária foi de 8 a 13 anos, de ambos os sexos. 0 Grupo 1 foi constituído por 20 participantes não obesos e o Grupo 2 por 20 participantes obesos. 0 público-alvo pertence à Escola de Educação Básica Professora Zélia Scharf, do município de Chapecó (SC).

Para a realização desta pesquisa, foi solicitada primeiramente a aprovação do Comitê de Ética em Pesquisas da Universidade Comunitária Regional de Chapecó (Unochapecó), a qual foi aprovada com protocolo n. 009/11.

Os critérios de inclusão foram a faixa etária entre 8 e 13 anos, de ambos os sexos. No Grupo 1, os participantes apresentaram Índice de Massa Corporal (IMC) dentro da normalidade. No Grupo 2, os participantes obtiveram IMC classificado como obesidade. Os critérios de exclusão referem-se a alguma doença associada, tanto crônica quanto aguda, ou ao responsável não assinar o Termo de Consentimento Livre e Esclarecido.

Para realizar a coleta de dados, foi encaminhado à Gerência Regional de Educação um termo solicitando a autorização da pesquisa. Após essa etapa, os responsáveis pelas crianças foram comunicados dos procedimentos e, se houve mútuo acordo entre crianças e responsáveis, o Termo de Consentimento Livre e Esclarecido foi assinado.

0 instrumento para coleta de dados utilizados foi questionário inicial, contendo dados sobre as medidas antropométricas - idade, gênero, peso, altura e Índice de Massa Corporal - e medidas específicas - pico máximo de fluxo expiratório (PEF), força da musculatura inspiratória (PI máx) e força da musculatura expiratória (PE máx). Os valores de referência do IMC utilizados na pesquisa constam na tabela 1 . 0 peso foi obtido na balança eletrônica Sunrise Personal e a altura medida por meio da fita métrica da marca ISP. As crianças permaneceram descalças e vestidas.

A avaliação do pico máximo de fluxo expiratório foi mensurado através do aparelho ASSESS ${ }^{\circledR}$ Full Range Peak Flow Meter (60-880L/min), da marca Respironics ${ }^{\circledR}$, com bucais descartáveis. As crianças permaneceram em pé e respirando profundamente, após realizaram apneia para que o aparelho fosse posicionado na boca e em seguida expiraram fortemente com a via aérea superior ocluída com clipe nasal. Na medição foram anotadas no mínimo três medidas satisfatórias de cada pico, ou seja, sem vazamento de ar pela boca ou pelo nariz e o maior valor obtido foi considerado para análise. Os valores de referência para medida do fluxo expiratório estão apresentados na tabela 2.

Logo após, os participantes foram submetidos à avaliação da força muscular respiratória por intermédio da manovacuometria, que mensura as pressões respiratórias máximas. Para a medição das pressões respiratórias máximas foi utilizado o manovacuômetro analógico da marca Comercial Médica ${ }^{\circledR}$, que possui mostrador com intervalos de escala de 4 em $4 \mathrm{cmH}_{2} 0$ e variação +/- $120 \mathrm{cmH}_{2} 0$.

Para a mensuração da PI máx, o indivíduo foi orientado a realizar uma expiração máxima, em nível de Volume Residual. Em seguida, o operador conectou o bucal e ocluiu a via aérea superior através do clipe nasal, solicitando que o indivíduo efetuasse um esforço inspiratório máximo, por via oral. Já para a medição da PE máx, o indivíduo realizou uma inspiração profunda, ao nível da Capacidade Pulmonar Total (CPT). 0 operador adaptou o bucal e, com a via aérea ocluída com o clipe nasal, 
solicitou ao indivíduo que efetuasse um esforço expiratório máximo, por via oral.

Após a coleta dos dados, os resultados foram organizados em tabelas com o objetivo de interpretar e comparar as medidas entre os dois grupos participantes da pesquisa. Para análise dos dados utilizou-se a média, desvio padrão e Teste t de Student, obtendo seus valores através do o sofware Bioestat 5.0 da Universidade Federal do Pará.

\section{Resultados e discussão}

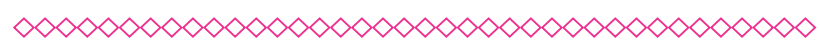

O grupo de crianças não obesas apresentou média de 10 anos; já de crianças obesas ficou entre 10 anos para meninas e 9 anos para meninos. Desta forma, não foi evidenciada diferença significativa quanto à média das idades das crianças avaliadas. A média da altura no grupo de obesos foi de $1,49 \mathrm{~m}$ e no grupo dos não obesos foi de $1,46 \mathrm{~m}$.

Obteve-se uma média maior quanto ao índice de massa corporal (IMC) de obesos do sexo masculino $(29,21)$, sendo o valor normal de 14,6 a 19,0; quanto ao sexo feminino, obteve-se uma média de 25,80 , sendo o valor de normalidade entre 14,5 a 20,7, conforme tabela 3.

Os dados encontrados são preocupantes, pois o início da obesidade ocorre ainda nas idades mais tênues do ser humano e persistir até a idade adulta. Assim, o diagnóstico precoce é de grande interesse para a saúde pública, pois o tratamento nesta fase inicial da vida pode ser mais eficiente e evitar complicações na vida adulta ${ }^{5}$.

Quanto à média do pico de fluxo expiratório no primeiro segundo, o grupo de crianças obesas obteve valor de $243,5 \mathrm{~L} / \mathrm{min}$, sendo o valor de normalidade $367,5 \mathrm{~L} / \mathrm{min}$. No grupo de crianças não obesas o valor na coleta foi de $342 \mathrm{~L} / \mathrm{min}$ e o valor de normalidade $352,5 \mathrm{~L} / \mathrm{min}$. Ou seja, o grupo de crianças não obesas do sexo masculino acima da média de valores recomendados para a sua altura, que é de $328 \mathrm{~L} / \mathrm{min}$, e o grupo de crianças do sexo feminino, levemente abaixo do esperado, apresentando no Teste $t$ de Student um valor de $p<0,0001$. Os valores da coleta de dados estão apresentados na tabela 4.

Os valores encontrados condizem com outras pesquisas que mostram que a obesidade reduz a função pulmonar, especialmente a capacidade vital (CV) e o volume expiratório forçado (VEF) ${ }^{9}$, aumentando a responsividade das vias aéreas e 0 trabalho elástico e resistivo da respiração, o que pode causar a dispneia ${ }^{10}$.

Também se obteve uma significativa diferença quanto a média dos valores de pressão inspiratória máxima comparando crianças obesas (101,5 $\left.\mathrm{CmH}_{2} \mathrm{O}\right)$ com as não obesas $\left(116 \mathrm{cmH}_{2} \mathrm{O}\right)$, pois estas obtiveram um valor relativamente maior, com valor estatístico no Teste t de Student de $p<0,006$, como pode-se observar na tabela 5.

Na média dos valores de pressão expiratória máximas apresentados na tabela 6 , as crianças obesas obtiveram valor de $84,5 \mathrm{cmH}_{2} \mathrm{O}$ e as crianças não obesas valor igual a $107 \mathrm{cmH}_{2} \mathrm{O}$. Diante da diferença, pode-se afirmar que as crianças obesas apresentaram redução da força muscular respiratória, principalmente da musculatura expiratória, com valores encontrados estatisticamente no Teste $t$ de Student de $p<0,0001$.

0 excesso de peso durante o crescimento pode gerar restrição pulmonar devido à diminuição da excursão diafragmática, causada pelo aumento da adiposidade abdominal ou pelo peso da parede torácica. Tal diminuição também pode estar associada à deposição de gordura nos músculos, principalmente nos abdominais, responsáveis pela força expiratória. Com isso, haveria alterações progressivas na função pulmonar que, associadas ao crescimento, desencadeariam modificações na composição das fibras musculares, tanto em sua qualidade quanto em sua capacidade oxidativa, reduzindo, dessa forma, a força muscular respiratória ${ }^{6}$.

Qualquer condição que aumente a carga de trabalho dos músculos respiratórios ou que leve a fraqueza são fatores predisponentes a fadiga muscular. 0 aumento de trabalho muscular, devido a um aumento de carga imposta, como ocorre em pessoas obesas, é uma condição predisponente quando submetido a uma determinada carga a qual é reversível pelo cansaço ${ }^{11}$.

Em análise estatística, usando o Teste t de Student, utilizado para analisar duas amostras populacionais pequenas e independentes, observa-se significância de resultados, com valor de $\mathrm{P}$ unilateral de pico de fluxo expiratório e pressão inspiratória máxima menor que 0,0001. E de pressão ex- 
piratória máxima menor que 0,0006. Esta análise possui intervalo de confiança de $95 \%$.

Apesar de analisada uma pequena população, se pode observar significância estatística dos resultados aqui apresentados, evidenciando assim uma diferença significativa quanto aos valores analisados, demonstrando que crianças obesas realmente apresentam um menor desempenho na capacidade respiratória quando comparadas a crianças não obesas.

Embora seja cada vez mais evidente que a obesidade leva ao aumento dos sintomas de dispneia, não é certo se a obstrução do fluxo aéreo é diretamente responsável por este sintoma em populações com sobrepeso e obesidade ${ }^{12}$.

Algumas alterações clássicas da função pulmonar provocadas pela obesidade podem ser observadas, como a redução do volume expiratório forçado no primeiro segundo, e as lacerações nos músculos respiratórios ${ }^{13}$.

O diafragma pode ter seu funcionamento prejudicado com a obesidade, provocando lesões diretas ao músculo ou a sua inervação. Pode também surgir, precocemente, doenças cardiovasculares, respiratórias, músculo-esqueléticas e metabólicas, ocasionando impacto na qualidade de vida dos indivíduos obesos².

Ainda encontra-se na literatura que crianças obesas são propensas a desenvolver hipoxemia, devido ao aumento da demanda ventilatória, aumento do esforço respiratório, diminuição da eficiência muscular e da reserva funcional, microectasias, infecções, asma e apneia do sono ${ }^{7}$. Isso não pôde ser evidenciado neste estudo, pois a avaliação traçada foi realizada de maneira que as crianças não atingissem esforços respiratórios extremos, e os sustentassem por algum período.

Os mecanismos pelos quais a obesidade promove essas disfunções respiratórias têm sido atribuídos a fatores mecânicos (volume abdominal) sobre a expansão pulmonar e as vias aéreas superiores ${ }^{14}$.

Nas faixas etárias estudadas em outras pesquisas, as crianças obesas apresentavam células adiposas maiores, atingindo o tamanho adulto até a idade de 11 anos. A entrada na vida adulta com um número baixo de células gordurosas deve garantir ao indivíduo uma vida livre de um quadro de obesidade extrema, visto que os adipócitos existentes não conseguiriam aumentar seu tamanho além de um determinado limite. Por outro lado, aqueles que iniciam a vida adulta com um número elevado de células adiposas estarão destinados a uma vida marcada pela obesidade ${ }^{15}$.

\section{Conclusão}

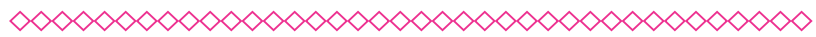

Com estes resultados, pode-se concluir que as crianças obesas apresentaram redução no pico máximo de fluxo expiratório e redução da força muscular inspiratória e expiratória. Diante disso, sugere-se que realizem pesquisas futuras, com uma maior quantidade de população de crianças obesas, já que a obesidade traz consequências sociais e psicológicas em potencial.

Além da obstrução, obesidade também afeta a mecânica respiratória, volumes pulmonares, diminui a função muscular e pulmonar, e aumenta o custo do trabalho e da energia da respiração ${ }^{16}$.

As pesquisas demonstram que metade dos pais e/ou responsáveis que têm filhos obesos não os consideram desta forma ${ }^{6}$. Isso pôde ser observado durante a realização deste trabalho, ocasionando uma grande preocupação, pois a não aceitação dos pais ou dos responsáveis gera dificuldade para o tratamento da obesidade infantil, em que a reeducação alimentar e o incentivo à vida saudável dependem da participação dos pais. Foi, ainda, observado que uma parcela da população de pais ou de responsáveis possui a mentalidade de que gordura é sinônimo de saúde.

O tratamento para a obesidade é igualmente complexo, tendo um atendimento multiprofissional prolongado, com auxílio de médicos, fisioterapeutas, nutricionistas e educadores físicos, visando modificações no comportamento e hábitos de vida, observando a criança de forma global. 0 tratamento busca uma reeducação alimentar, restauração da função pulmonar e da sua musculatura, inserindo uma atividade física regular, com auxílio fundamental e insubstituível da estrutura familiar. Assim, o objetivo básico é manter o peso adequado para a estatura, preservando o crescimento e 0 desenvolvimento normais da criança. 
A atividade física, mesmo que espontânea, é importante na composição corporal, por aumentar a massa óssea e prevenir a osteoporose e a obesidade $^{15}$.

O fisioterapeuta tem sob sua responsabilidade principal manter as conduções clínicas ideais da função muscular respiratória em qualquer etapa ou fase da terapia. O reconhecimento dos componentes restritivos e obstrutivos dos quais o paciente com disfunção muscular respiratória pode ser portador é absolutamente importante para o acompanhamento terapêutico. Ele pode ser obtido de uma forma simples, utilizando um conjunto de equipamentos, destacando-se o Peak Flow ${ }^{17}$.

\section{Referências}

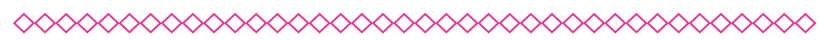

1. Filho GMC, Filho ADR, Ribeiro GCC. Provas de função pulmonar no pré e pós-operatório de redução gástrica por celiotomia ou por videolaparoscopia. Rev. Col. Bras. 2008;35(6).

2. Scodeler NF, Paschoal MA. Avaliação da capacidade funcional cardiorrespiratória de crianças obesas e não obesas sedentárias. In: Anais do XIII Encontro de Iniciação Científica da PUC-Campinas; 2008.

3. Rodrigues PPC. Estudo da relação entre o índice da massa corporal (IMC) e a postura corporal em estudantes do ensino fundamental da Escola São Judas Tadeu, Tubarão SC. Universidade do sul de Santa Catarina. Tubarão, 2007.

4. Bueno MB, Ferberg RM. Comparação de três critérios de classificação de sobrepeso e obesidade entre pré-escolar. Rev. Bras. Saúde Matern. Infant. 2006.

5. Bouchard C. Atividade Física e Obesidade. 1. ed. Barueri: Manole, 2003.

6. Santiago SQ, Silva MLP, Davidson J, Aristóteles LRCRB. Avaliação da força muscular respiratória em crianças e adolescentes com sobrepeso/ obesos. Rev. paul. Pediatr. 2008;26(2).
7. Mello ED, Luft, VC, Meyer F. Obesidade infantil: como podemos ser eficazes? Jornal de pediatria. 2004; 80(3).

8. Leite JM. Obesidade infantil e alterações das provas funcionais respiratórias [dissertação de mestrado], 2009.

9. Schachter LM, Salome CM, Peat JK, Woolcock AJ. Obesity is a risk for asthma and wheeze but not airmay hyperresponsiveness. Thorax. 2001;56:4-8.

10. Salome CM. Efeito da obesidade sobre a falta de ar e responsividade das vias aéreas à metacolina em não asmático. International Journal of Obesity. 2008.

11. Gambaroto G. Fisioterapia Respiratória em Unidade de Terapia Intensiva. São Paulo: Ateneu, 2006.

12. Sin DD, Jones RL, Man SF. Obesity Is a Risk Factor for Dyspnea but Not for Airflow Obstruction. Archives of Internal Medicine. 2002; 162(13).

13. Zamboni M, Pereira CAC. Pneumologia: diagnóstico e tratamento. São Paulo: Atheneu, 2006.

14. Dâmaso A. Obesidade. Rio de Janeiro: Guanabara Koogan, 2003.

15. Pollock ML, Rocha ML, Wilmore JH. Exercícios na saúde e na doença: avaliação e prescrição para prevenção e reabilitação. 2. ed. Rio de Janeiro: MEDSI, 1993.

16. Soares LD, Petroski, EL. Prevalência, fatores etiológicos e tratamento da obesidade infantil. Rev. Bras. 2003;5(1).

17. Azeredo CAC. Fisioterapia respiratória moderna. 4. ed. ampl. e rev. São Paulo: Manole, 2002. 


\section{Anexos}

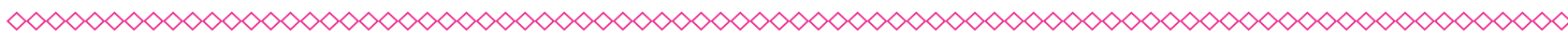

Tabela 1 - Valores do Índice de Massa Corporal infantil

\begin{tabular}{|c|c|c|c|c|}
\hline Idade & Sexo & Normal & Sobrepeso & Obesidade \\
\hline \multirow{2}{*}{6} & Masculino & $14,1-17,2$ & $17,2-18,8$ & Acima de 18,0 \\
\hline & Feminino & $13,7-17,0$ & $17,0-17,5$ & Acima de 17,5 \\
\hline \multirow{2}{*}{7} & Masculino & $14,4-17,5$ & $17,5-18,2$ & Acima de 18,2 \\
\hline & Feminino & $14,1-17,5$ & $17,5-18,3$ & Acima de 18,3 \\
\hline \multirow{2}{*}{8} & Masculino & $14,3-18,0$ & $18,0-19,1$ & Acima de 19,1 \\
\hline & Feminino & $14,1-18,7$ & $18,7-19,8$ & Acima de 19,8 \\
\hline \multirow{2}{*}{9} & Masculino & $14,6-19,0$ & $19,0-19,9$ & Acima de 19,9 \\
\hline & Feminino & $14,6-19,8$ & $19,8-21,2$ & Acima de 21,2 \\
\hline \multirow{2}{*}{10} & Masculino & $15,0-19,8$ & $19,8-19,8$ & Acima de 19,8 \\
\hline & Feminino & $14,5-20,7$ & $20,7-22,0$ & Acima de 22,0 \\
\hline \multirow{2}{*}{11} & Masculino & $15,1-21,5$ & $21,5-22,5$ & Acima de 22,5 \\
\hline & Feminino & $15,3-21,8$ & $21,8-23,4$ & Acima de 23,4 \\
\hline \multirow{2}{*}{12} & Masculino & $15,7-21,7$ & $21,7-23,7$ & Acima de 23,7 \\
\hline & Feminino & $15,6-23,1$ & $23,1-24,6$ & Acima de 24,6 \\
\hline \multirow{2}{*}{13} & Masculino & $16,4-22,2$ & $22,2-24,0$ & Acima de 24,0 \\
\hline & Feminino & $16,3-23,8$ & $23,8-25,2$ & Acima de 25,2 \\
\hline
\end{tabular}

Fonte: Organização Mundial da Saúde (OMS).

Tabela 2 - Padrões normais do Pico de Fluxo Expiratório

$\begin{array}{cccc}\begin{array}{c}\text { Estatura } \\ (\mathrm{cm})\end{array} & \begin{array}{c}\text { Valor } \\ (\mathrm{L} / \mathrm{min})\end{array} & \begin{array}{c}\text { Estatura } \\ (\mathrm{cm})\end{array} & \begin{array}{c}\text { Valor } \\ (\mathrm{L} / \mathrm{min})\end{array} \\ 109 & 145 & 142 & 328 \\ 112 & 169 & 145 & 344 \\ 114 & 180 & 147 & 355 \\ 117 & 196 & 150 & 370 \\ 119 & 207 & 152 & 381 \\ 122 & 222 & 155 & 397 \\ 124 & 233 & 157 & 407 \\ 127 & 249 & 160 & 423 \\ 130 & 265 & 163 & 439 \\ 135 & 291 & 165 & 450 \\ 137 & 302 & 168 & 466 \\ 140 & 318 & 170 & 476\end{array}$

Fonte: Sarmento (apud Godfrey et al., 1970).

(clique para voltar ao texto) 
Tabela 3 - Média das medidas antropométricas

\begin{tabular}{lcccccccc} 
Grupo & \multicolumn{1}{c}{ Sexo (\%) } & \multicolumn{1}{c}{ Idade (\%) } & \multicolumn{1}{c}{ Média } & IMC & \multicolumn{3}{c}{ Altura (m) } \\
& F & M & F & M & F & M & F & M \\
Obesos & 45 & 55 & 10,11 & 10,09 & 25,80 & 29,21 & 1,50 & 1,48 \\
Não obesos & 70 & 30 & 10,08 & 9,50 & 17,04 & 16,53 & 1,50 & 1,42 \\
\hline
\end{tabular}

$\mathrm{F}=$ Feminino; $\mathrm{M}=$ Masculino.

Fonte: elaboração das autoras.

(clique para voltar ao texto)

Tabela 4 - Média do Pico Máximo de Fluxo Expiratório

\begin{tabular}{lcc} 
Peak Flow & Média (L/min) & Desvio padrão \\
Não obesos & 342 & 47,08 \\
Obesos & 243,5 & 62,85 \\
\hline
\end{tabular}

Teste t: 5,60; $p$ (unilateral): $<0,0001$.

Fonte: elaboração das autoras.

(clique para voltar ao texto)

Tabela 5 - Média da Pressão inspiratória máxima

\begin{tabular}{lcc} 
PI Max & Média $\left(\mathrm{cmH}_{2} \mathrm{O}\right)$ & Desvio padrão \\
Grupo não obeso & 116 & 8,20 \\
Grupo obeso & 101,5 & 15,98 \\
\hline
\end{tabular}

Teste t: 3,60; $\mathrm{p}$ (unilateral): $<0,0006$.

Fonte: elaboração das autoras.

(clique para voltar ao texto)

Tabela 6 - Média da Pressão expiratória máxima

\begin{tabular}{lcc} 
PE Max & Média $\left(\mathrm{cmH}_{2} \mathrm{O}\right)$ & Desvio padrão \\
Grupo não obeso & 107 & 10,31 \\
Grupo obeso & 84,5 & 9,99 \\
\hline
\end{tabular}

Teste t: 7,00; p (unilateral): <0,0001.

Fonte: elaboração das autoras.

(clique para voltar ao texto) 